\title{
PHYSICS AND MATHEMATICS
}

\section{POWER GEOMETRY IN LOCAL RESOLUTION OF SINGULARITIES OF AN ALGEBRAIC CURVE}

\author{
Akhmadjon Soleev \\ Doctor of science, professor Samarkand State University, Uzbekistan, \\ ORCID ID: https://orcid.org/0000-0003-4921-2349
}

DOI: https://doi.org/10.31435/rsglobal_ws/30062020/7100

\section{ARTICLE INFO}

Received: 25 April 2020

Accepted: 10 June 2020

Published: 30 June 2020

\section{KEYWORDS}

power geometry, space curve, singular point, Newton polyhedron, normal cone, power transformations.

MSC (2010): 14B05, 53A04,

58E07, 58K45, 58K55.

\begin{abstract}
The main goal of this work is to provide a consistent set of generalpurpose algorithms for analyzing singularities applicable to all types of equations. We present the main ideas and algorithms of power geometry and give an overview of some of its applications. We also present a procedure that allows us to distinguish all branches of a spatial curve near a singular point and calculate the parametric appearance of these branches with any degree of accuracy. For a specific case, we show how this algorithm works.
\end{abstract}

Citation: Akhmadjon Soleev. (2020) Power Geometry in Local Resolution of Singularities of an Algebraic Curve. World Science. 6(58), Vol.1. doi: 10.31435/rsglobal_ws/30062020/7100

Copyright: (C) 2020 Akhmadjon Soleev. This is an open-access article distributed under the terms of the Creative Commons Attribution License (CC BY). The use, distribution or reproduction in other forums is permitted, provided the original author(s) or licensor are credited and that the original publication in this journal is cited, in accordance with accepted academic practice. No use, distribution or reproduction is permitted which does not comply with these terms.

Introduction. Many problems in mathematics, physics, biology, economics, and other sciences are reduced to nonlinear equations or systems of such equations. The equations may be algebraic, ordinary differential or partial differential and systems may comprise the equations of one type, but may include equations of different types. The solutions of these equations and systems subdivide into regular and singular ones. Near a regular solution, the implicit function theorem or its analogs are applicable, which gives a description of all neighboring solutions. Near a singular solution, the implicit function theorem is inapplicable, and until recently, there had been no general approach to the analysis of solutions neighboring the singular one. Although different methods of such analysis were suggested for some special problems.

Main Part. We develop a new calculus based on Power Geometry [1, 2, 3, 4]. Here we will consider only to compute local and asymptotic expansions of solutions to nonlinear equations of algebraic classes as well as to systems of such equations. But it can also be extended to other classes of nonlinear equations for such as differential, functional, integral, and integro-differential [7].

Ideas and algorithms are common for all classes of equations. Computation of asymptotic expansions of solutions consists of 3 following steps (we describe them for one equation $f=0$ ).

1. Isolation of truncated equations $\hat{f}_{j}^{(d)}=0$ by means of generalized faces of the convex polyhedron $\Gamma(f)$, which is a generalization of the Newton polyhedron. The first term of the expansion of a solution to the initial equation $f=0$ is a solution to the corresponding truncated equation $\hat{f}_{j}^{(d)}=0$.

2. Finding solutions to a truncated equation $\hat{f}_{j}^{(d)}=0$, which is quasi homogenous. Using power and logarithmic transformations of coordinates we can reduce the equation $\hat{f}_{j}^{(d)}=0$ to such a 
simple form that can be solved. Among the solutions found we must select appropriate ones that give the first terms of asymptotic expansions.

3. Computation of the tail of the asymptotic expansion. Each term in the expansion is a solution of a linear equation that can be written down and solved.

Elements of plane Power Geometry were proposed by Newton for algebraic equations (1670). Space Power Geometry for a nonlinear autonomous system of ODEs was proposed by Bruno (1962) [1]. Thus, now it is exactly 50 years for the Newton polyhedron.

It is clear that this calculus cannot be mastered during this paper. We will try to summarize our ideas and in the next paper, we will consider this problem and give algorithms for nonlinear systems of algebraic equations.

\section{Algebraic equations [2,3].}

In this paper, we consider a polynomial depending on three variables near its singular point where the polynomial vanishes with all its first partial derivatives. We propose a method of computation of asymptotic expansions of all branches of the set of roots of the polynomial near the mentioned singular point. Now there are three types of expansions. The method of computation Is based on space Power Geometry. All examples are for polynomials in two variables.

Let $\boldsymbol{X}=\left(x_{1}, x_{2}, x_{3}\right) \subset R^{3}$ or $C^{3}$ and $f(\boldsymbol{X})$ be a polynomial. $\boldsymbol{X}^{0}$ is called to be singular for the set $\mathcal{F}=\{\boldsymbol{X}: f(\boldsymbol{X})=0\}$ if all the partial derivatives of the first order of the polynomial $f$ vanish at the point $\boldsymbol{X}^{0}$ and $f\left(\boldsymbol{X}^{0}\right)=0$.

Consider the following problem. Near the singular point $\boldsymbol{X}^{0}$ for each branch of the set $\mathcal{F}$, find a parameter expansion of one of the following three types [6].

Type 1

$$
x_{1}=\sum_{k=1}^{\infty} b_{k} v^{k}, \quad x_{2}=\sum_{k=1}^{\infty} c_{k} v^{k}, \quad x_{3}=\sum_{k=1}^{\infty} d_{k} v^{k},
$$

where $b_{k}, c_{k}, d_{k}$ are constants.

Type 2

$$
x_{1}=\sum b_{p q} u^{p} v^{q}, \quad x_{2}=\sum c_{p q} u^{p} v^{q}, \quad x_{3}=\sum d_{p q} u^{p} v^{q},
$$

where $b_{p q}, \mathrm{c}_{p q}, d_{p q}$ are constants and integer points $(p, q)$ are in a sector with the angle less than $\pi$.

Type 3

$$
x_{1}=\sum_{k=0}^{\infty} \beta_{k}(u) v^{k}, \quad x_{2}=\sum_{k=0}^{\infty} \gamma_{k}(u) v^{k}, \quad x_{3}=\sum_{k=0}^{\infty} \delta_{k}(u) v^{k},
$$

where $\beta_{k}(u), \gamma_{k}(u), \delta_{k}(u)$ are rational functions of $u$ and $\sqrt{\psi(u)}$, and $\psi(u)$ is a polynomial in $u$.

Objects and algorithms of Power Geometry.

Let a finite sum be given (for example, a polynomial)

$$
f(\boldsymbol{X})=\sum f_{Q} \boldsymbol{X}^{Q} \text { over } Q \in S \text {, }
$$

where $\boldsymbol{X}=\left(x_{1}, x_{2}, x_{3}\right) \in \mathbb{R}^{3}, Q=\left(q_{1}, q_{2}, q_{3}\right) \in \mathbb{R}^{3}$ and $\boldsymbol{X}^{Q}=x_{1}^{q_{1}} x_{2}^{q_{2}} x_{3}^{q_{3}}, f_{Q}=$ const $\epsilon \mathbb{R}$.

To each of the summand of $f$ the sum (4.1), we assign it vector power exponent $Q$, and to the whole sum (4.1), we assign the set of all vector power exponents of its terms, which is called the support of the sum (4.1) or of the polynomial $f(X)$, and it is denoted by $S(f)$. The convex hull of the support $S(f)$ is called the Newton polyhedron of the $\operatorname{sum} f(X)$ and it is denoted by $\Gamma(f)$.

The boundary $\partial \Gamma$ of the polyhedron $\Gamma(f)$ consists of generalized faces $\Gamma_{j}^{(d)}$ of various dimensions $d=0,1,2$. Here $j$ is the number of a face. To each generalized face $\Gamma_{j}^{(d)}$, we assign the truncated $\operatorname{sum} \hat{f}_{j}^{(d)}(\boldsymbol{X})=\sum f_{Q} \boldsymbol{X}^{Q}$ over $Q \in \Gamma_{j}^{(d)} \cap S(f)$.

Example 1. We consider the polynomial $f(x, y)=\boldsymbol{x}^{\mathbf{5}}+\boldsymbol{y}^{\mathbf{5}}-\boldsymbol{x} \boldsymbol{y}^{\mathbf{2}}$. Support $\mathbf{S}(f)$ consists from points $Q_{1}=(5,0), Q_{2}=(0,5), Q_{3}=(1,2)$.

The Newton polygon $\Gamma(f)$ is the triangle $Q_{1} Q_{2} Q_{3}$ (figure 1). Edges and corresponding truncated polynomials are

$$
\Gamma_{1}^{(1)}: \hat{f}_{1}^{(1)}=x^{5}-x y^{2}, \quad \Gamma_{2}^{(1)}: \hat{f}_{2}^{(1)}=y^{5}-x y^{2}, \quad \Gamma_{3}^{(1)}: \hat{f}_{3}^{(1)}=x^{5}+y^{5},
$$
product

Let $\mathbb{R}_{*}^{3}$ be a space dual to space $\mathbb{R}^{3}$ and $S=\left(s_{1}, s_{2}, s_{3}\right)$ be points of this dual space. The scalar 


$$
\langle Q, S\rangle=q_{1} s_{1}+q_{2} s_{2}+q_{3} s_{3}
$$

is defined for the points $Q \in \mathbb{R}^{3}$ and $S \in \mathbb{R}_{*}^{3}$. Specifically, the normal external $N_{k}$ to the generalized face $\Gamma_{k}^{(d)}$ is a point in $\mathbb{R}_{*}^{3}$.

The scalar product $\left\langle Q, N_{k}\right\rangle$ reaches the maximum value at the points $Q \epsilon \Gamma_{k}^{(d)} \cap S$, i.e., at the points of the generalized face $\Gamma_{k}^{(d)}$. Moreover, set of all points $S \in \mathbb{R}_{*}^{3}$, at which the scalar product (4.2) reaches the maximum over $Q \in \boldsymbol{S}(f)$ exactly at points $Q \in \Gamma_{k}^{(d)}$, is called the normal cone of the generalized face $\Gamma_{k}^{(d)}$ and is denoted by $\boldsymbol{U}_{k}^{(d)}$.

Example 2: (cont. of Example 1). For faces $\Gamma_{k}^{(d)}$ of the Newton polygon $\Gamma(f)$ of Figure 1, normal cones are shown in Figure 2.

For edge $\Gamma_{j}^{(1)}{ }_{j}=1,2,3$ normal cone $U_{j}^{(1)}$ is a ray orthogonal to its edge. For vertex $\Gamma_{j}^{(0)}=Q_{j}=$ $R_{j}, j=1,2,3$ normal cone is open sector between rays orthogonal to edges $\Gamma_{j}^{(1)}$ adjacent to vertex $R_{j}$.

Theorem 1. If for $t \rightarrow \infty$ the curve

$$
x_{1}=b t^{s_{1}}(1+(0)), x_{2}=c t^{s_{2}}(1+(0)), x_{3}=d t^{s_{3}}(1+(0))
$$

where $b, c, d$ and $s_{i}$ are constants, belongs to the set $\Omega$, and the vector $S=\left(s_{1}, s_{2}, s_{3}\right) \in \mathbf{U}_{k}^{(d)}$, then the first approximation $\mathrm{x}_{1}=\mathrm{bt}^{\mathrm{s}_{1}}, \mathrm{x}_{2}=\mathrm{ct}^{\mathrm{s}_{2}}, \mathrm{x}_{3}=\mathrm{dt}^{\mathrm{s}_{3}}$ of the curve (4.3) satisfies the truncated equation $\hat{\mathrm{f}}_{\mathrm{k}}^{(\mathrm{d})}(\mathbf{X})=0$.

See the proof of the theorem in the paper [2,3].

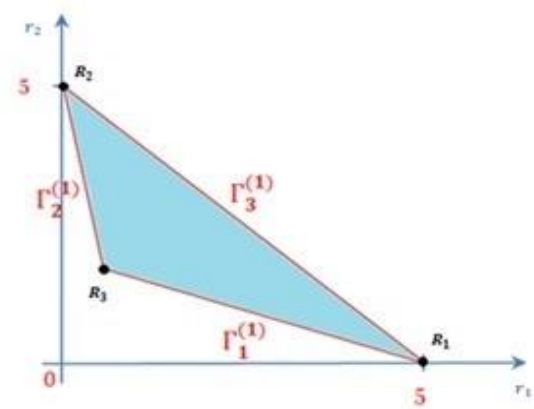

Fig. 1

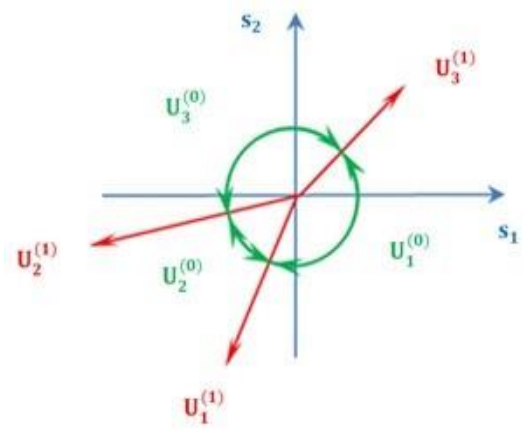

Fig. 2

The truncated sum $\hat{f}_{j}^{(0)}$ corresponding to the vertex $\Gamma_{j}^{(0)}$ is a monomial. Such truncations are of no interest and will not be considered. We will consider truncated sums corresponding to edges $\Gamma_{j}^{(1)}$ and faces $\Gamma_{j}^{(2)}$ only.

Power transformations have the form

$$
\log \boldsymbol{X}=\boldsymbol{\alpha} \log \boldsymbol{Y}
$$

where $\log X=\left(\log x_{1}, \log x_{2}, \log x_{3}\right)^{T}, \log Y=\left(\log y_{1}, \log y_{2}, \log y_{3}\right)^{T}, \quad \alpha \quad$ is a nondegenerate square $3 \times 3$ matrix $\left(\alpha_{i j}\right)$ with rational elements $\alpha_{i j}$ (they are often integer).

The monomial $\boldsymbol{X}^{Q}$ is transformed to the monomial $\boldsymbol{Y}^{Q_{1}}$ by the power transformation (4.4), where $Q_{1}^{T}=\boldsymbol{\alpha}^{T} Q^{T}$. Power transformations and multiplications of a polynomial by monomial generate the affine geometry in space $\mathbb{R}^{3}$ of vector power exponents of polynomial monomials. The matrix $\alpha$ with integer elements and det $\boldsymbol{\alpha}= \pm 1$ is called unimodular.

Theorem 2. For the face $\Gamma_{j}^{(d)}$, there exists a power transformation (4.4) with a unimodular matrix $\boldsymbol{\alpha}$ which transforms the truncated sum $\hat{f}_{j}^{(d)}(\boldsymbol{X})$ into the sum in d coordinates, i.e. $\hat{f}_{j}^{(d)}(\boldsymbol{X})=Y^{Q^{\prime}} h(\boldsymbol{Y})$, where $h(\boldsymbol{Y})=h\left(y_{1}\right)$ if $d=0, h(\boldsymbol{Y})=h\left(y_{2} y_{3}\right)$ if $d=2$. Here $Q^{\prime}=q_{1}^{\prime}, q_{2}^{\prime}, q_{3}^{\prime} \in \mathbb{R}^{3}$ and other coordinates $y_{2}, y_{3}$ for $d=1$, for $d=2$ are small. For the polynomial $\hat{f}_{j}^{(d)}(\boldsymbol{X})$ the sum $h(\boldsymbol{Y})$ is also polynomial.

The proof of this theorem is similar to the proof of theorem 3 in the paper [2].

The cone of the problem $\boldsymbol{K}$ is a set of such vectors $S=\left(s_{1}, s_{2}, s_{3}\right) \in \mathbb{R}_{*}^{3}$ that curves of the form (4.3) fill the part of the space $\left(x_{1}, x_{2}, x_{3}\right)$, which must be studied. So, our initial problem corresponds 
to the cone of the problem $K=\left\{S=\left(s_{1}, s_{2}, s_{3}\right): S<0\right\}$ in $\mathbb{R}_{*}^{3}$, because $x_{1}, x_{2}, x_{3} \rightarrow 0$. If $x_{1} \rightarrow \infty$ then $s_{1}>0$ in the cone of the problem $\boldsymbol{K}$.

Example 3. For variables $\boldsymbol{x}, \boldsymbol{y}$ near origin $\boldsymbol{x}=\boldsymbol{y}=\mathbf{0}$ cone of the problem is the quadrant III: $\left\{\boldsymbol{K}_{\mathbf{3}}=s_{1} \boldsymbol{s}_{\mathbf{2}}>0\right\}$. In Figure 2 some cones of the problem $\boldsymbol{K}_{i}$ intersects several normal cones $\boldsymbol{U}_{\boldsymbol{j}}^{(2)}$. E.g. $K_{3}$ intersects $U_{\mathbf{1}}^{(\mathbf{1})}, U_{\mathbf{2}}^{(\mathbf{1})}$ and $U_{\mathbf{1}}^{(\mathbf{0})}, \boldsymbol{U}_{\mathbf{2}}^{(\mathbf{0})}, \boldsymbol{U}_{\mathbf{3}}^{(\mathbf{0})}$. $\boldsymbol{K}_{\mathbf{l}}$ intersects $\boldsymbol{U}_{\mathbf{3}}^{(\mathbf{1})}, \boldsymbol{U}_{\mathbf{1}}^{(\mathbf{0})}, \boldsymbol{U}_{\mathbf{2}}^{(\mathbf{0})}$.

Let's give a step-by-step algorithm for solving the problem.

1. We compute the support $S(f)$, the Newton polyhedron $\Gamma(f)$, its two-dimensional faces $\Gamma_{j}^{(2)}$ and their external normal $N_{j}$. Using normal $N_{j}$ we compute the normal cones $U_{k}^{1}$ to edges $\Gamma_{k}^{(1)}$.

2. We select all the edges $\Gamma_{k}^{(1)}$ and faces $\Gamma_{j}^{(2)}$, which normal cones intersect the cone of the problem $K$. It is enough to select all the faces $\Gamma_{j}^{(2)}$, which external normal $N_{j}$ intersect the cone of the problem $\boldsymbol{K}$, and then add all the edges $\Gamma_{k}^{(1)}$ of these faces

a) For each of the selected edge $\Gamma_{k}^{(1)}$, we fulfill a power transformation $\boldsymbol{X} \rightarrow \boldsymbol{Y}$ of Theorem 2 and we get the truncated equation in a form $h\left(y_{1}\right)=0$.

b) We find its roots. Let $y_{1}^{0}$ be one of its roots.

c) We fulfill the power transformation $\boldsymbol{X} \rightarrow \boldsymbol{Y}$ in the whole polynomial $f(\boldsymbol{X})$ and we get the polynomial $f_{1}(\boldsymbol{Y})$.

d) We make the shift $z_{1}=y_{1}-y_{1}^{0}, z_{2}=y_{2}, z_{3}=y_{3}$ in the polynomial $f_{1}(\boldsymbol{Y})$ and get the polynomial $f_{2}(\boldsymbol{Z})$.

3. If $y_{1}^{0}$ is a simple root of the equation $h\left(y_{1}\right)=\mathbf{0}$ then, according to Implicit Function Theorem, it corresponds to an expansion of the form $\boldsymbol{y}_{2}=\sum \boldsymbol{a}_{p q} \boldsymbol{y}_{1}^{p} \boldsymbol{y}_{3}^{q}$ where $\boldsymbol{a}_{p q}$ are constants. It gives an expansion of type 2 in coordinates $\boldsymbol{Y}$.

4. For each of the selected face $\Gamma_{\boldsymbol{k}}^{(\mathbf{1})}$, we fulfill a power transformation $\boldsymbol{X} \rightarrow \boldsymbol{Y}$ of Theorem 2 and we get a truncated equation in the form $\widehat{\boldsymbol{h}}\left(\boldsymbol{y}_{1}, \boldsymbol{y}_{2}\right)=\mathbf{0}$. We factorize $\widehat{\boldsymbol{h}}\left(\boldsymbol{y}_{1}, \boldsymbol{y}_{2}\right)=\mathbf{0}$ into prime factors. Let $\tilde{h}\left(y_{1}, y_{2}\right)=0$ be one of such factors and its algebraic curve has genus $\rho$.

5. If $\rho=0$ then there exists birational uniformization $y_{1}=\xi\left(z_{2}\right), y_{2}=\eta\left(z_{2}\right)$ of this curve. We change variables $y_{1}=\xi\left(z_{2}\right)+z_{1}, y_{2}=\eta\left(z_{2}\right)$ and then $h$ is divided by $z_{1}$. We change variables in the whole polynomial $f(X)$ and get the polynomial $f_{2}(Z) \stackrel{\text { def }}{=} f_{1}(Y)=f(X)$

If $\tilde{h}\left(y_{1}, y_{2}\right)$ is a simple factor of $h\left(y_{1}, y_{2}\right)$ then roots of the polynomial $f_{2}(Z)$ are expanded into series of the form

$$
z_{1}=\sum_{k=1}^{\infty} a_{k}\left(z_{2}\right) z_{3}^{k}
$$

where $a_{k}\left(z_{2}\right)$ are rational functions of $z_{2}$. It gives an expansion of type 3 in original coordinates $\boldsymbol{X}$.

If $\tilde{h}\left(y_{1}, y_{2}\right)$ is a multiple factor of $h\left(y_{1}, y_{2}\right)$ then we compute the Newton polyhedron of the polynomial $f_{2}(Z)$, compute the cone of problem $\boldsymbol{K}_{2}=\left\{S: s_{2}, s_{3}<0\right\}$ and continue computations.

6. If $\rho=1$ (elliptic curve), there exists the birational change of variables $y_{1}, y_{2} \rightarrow z_{1}, z_{2}$, transforming $\tilde{h}\left(y_{1}, y_{2}\right)=0$ into the normal form $z_{1}^{2}-\psi\left(y_{2}\right)$, where $\psi$ is a polynomial of order 3 or 4 .

If $\rho>1$, we distinguish hyper-elliptic and non hyper-elliptic curves. The hyper-elliptic curve is birationally equivalent $y_{1}, y_{2} \rightarrow z_{1}, z_{2}$ to its normal form $z_{1}^{2}-\psi\left(y_{2}\right)$, where $\psi$ is a polynomial of order $2 \rho+1$ or $2 \rho+2$.

If factor $\tilde{h}$ of $h$ is simple we get expansions of solutions of equation $f_{2}(Z)=0$ into series (4.5), where $a_{k}$ are rational functions of $z_{2}$ and $\sqrt{\psi\left(z_{2}\right)}$. We get the expansion of type 3 in original coordinates $\boldsymbol{X}$.

If $\tilde{h}\left(y_{1}, y_{2}\right)$ is a multiple factor of $h\left(y_{1}, y_{2}\right)$ then we continue computation for $f_{2}(\boldsymbol{Z})$ as above.

In this procedure, we distinguish two cases:

1. Truncated polynomial contains a linear part of one of the variables. The generalization of the Implicit Function Theorem is applicable and it is possible to compute parametric expansion of a set of roots of a full polynomial.

2. Truncated polynomial does not contain a linear part of any variable. Then the Newton polyhedron for a full polynomial must be built and we must consider new truncated polynomials taking into account the new cone of the problem $\boldsymbol{K}$. 


\section{Example 4 (cont. of Examples 1-3).}

1. For edge $\Gamma_{1}^{(1)}$, we get a truncated equation $x^{5}-x y^{2}=0$ i.e. $y= \pm x^{2}$. It is case 1 , and this asymptotic form is continued into power expansion of branch

$$
y= \pm x^{2}+\sum_{k=2}^{\infty} b_{k} x^{2 k}
$$

near the origin $x=y=0$ (figure 3).

2. For edge $\Gamma_{2}^{(1)}$, we get a truncated equation $y^{5}-x y^{2}=0$ i.e. $y= \pm x^{1 / 3}$. It is case 1 , and these asymptotic forms are continued into power expansion branches

$$
y= \pm \sqrt[3]{x}+\sum_{k=2}^{\infty} b_{k} x^{\frac{k}{3}}
$$

near the origin $x=y=0$ (figure 4).

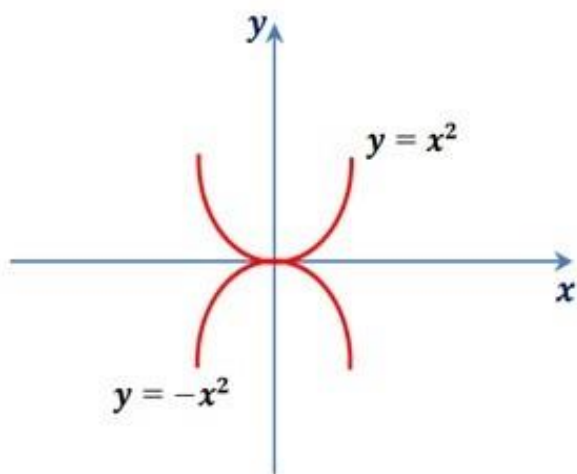

Fig. 3

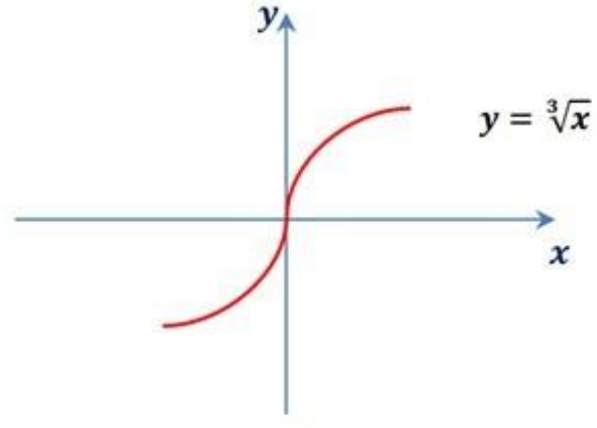

Fig. 4

3. For edge $\Gamma_{3}^{(1)}$, we get truncated equation $x^{3}+y^{3}=0$. It has the simple factor $x+y=0$, i.e $y=-x$. It is case 1 , and the power expansion at infinity is

$$
y=-x+\sum_{k=2}^{\infty} b_{k} x^{-k}
$$

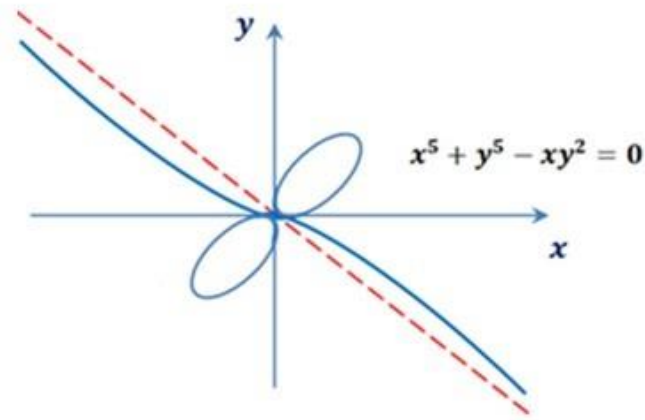

Fig. 5

Figure 5 shows a general view of the equation $f(x, y)=x^{5}+y^{5}-x y^{2}$. In the neighborhood of a singular point.

Asymptotic description of a subset of singular points of $\Omega$ can be obtained by the same procedure, but we have to select only singular points in each truncated equation. As a result, we obtain expansions of type one.

So we got the following result: If we perform calculations for 1-4 using this procedure, then at each step we find all the roots of the corresponding truncated equations, and find all the curves of the roots of the truncated equations with a positive native elliptic or hyperelliptic, we get a local description of each component of the set $\Omega$ adjacent to the starting point $\mathbf{X}^{0}$, in the form of expansions of types 1-3. 


\section{REFERENCES}

1. A.D. Bruno, Power Geometry in Algebraic and Differential Equations, North-Holland Mathematical Library, N, V.57, Elsevier, 2000.

2. A.D. Bruno, A.S. Soleev, Local uniformization of branches of a space curve and Newton polyhedra. Algebra and Analiz, 1991. Vol. 3, no. 1. P. 67-102.

3. A.S. Soleev, Algorithm of local resolution of singularities of a space curve, LNCS 3718, pp.405-415. Springer-Verlag, 2005.

4. A.S. Soleev, N.A.Soleeva Power Geometry for Finding Periodic Solutions in One System of ODE. Malaysian Journal of Mathematical Sciences, No 2, 2014.

5. A.S. Soleev, Singling out branches of an algebraic curve and Newton polyhedra, Dokl. Akad. Nauk SSSR 268 (1983), 1305-1307; (R) = Soviet Math Dokl. 27 (1983) (E).

6. A.D. Bruno, A.B. Batkhin Asymptotic solution of an algebraic equation, DAN 440:3 (2011) 295-300 (R) = Doklady Mathematics 84:2 (2011) 634639 (E).

7. Soleev A. Complicated Bifurcations of Periodic Solutions in some System of ODE. Canadian Mathem. Bulletin. Vol.39(3), 1996.p.360-366. 